\title{
FATE OF THE ANTEGRADE URETERIC STENT
}

\author{
Jerry A. Raju, BM BCh, FRCS (Urol) ${ }^{1,3}$, Cherian George, FRCR ${ }^{2}$, Prashant Patel, FRCS (Urol) ${ }^{3}$, \\ Samson Liu, MB ChB, FRCS, FRCS (Urol) ${ }^{1}$ \\ ${ }^{1}$ Department of Urology, Royal Stoke University Hospital, University Hospitals of North Midlands NHS \\ Trust, Stoke-on-Trent, United Kingdom \\ ${ }^{2}$ Imaging Department, Royal Stoke University Hospital, University Hospitals of North Midlands NHS Trust, \\ Stoke-on-Trent, United Kingdom \\ ${ }^{3}$ Department of Urology, Queen Elizabeth Hospital Birmingham, University Hospitals Birmingham NHS \\ Foundation Trust, Birmingham, United Kingdom
}

Corresponding Author Jerry A. Raju: j.raju@nhs.net

Submitted: July 8, 2018. Accepted: September 28, 2018. Published: November 13, 2018.

\begin{abstract}
Antegrade stents are commonly used to relieve malignant and benign ureteric obstruction. However, follow up of these patients often involves several specialties and the potential for delayed management and forgotten stents. This observational study reviews indications and outcomes of antegrade stent procedures at one university hospital to provide prognostic and quality improvement data.
\end{abstract}

\section{Patients and Methods}

A retrospective analysis of 152 antegrade stent procedures in 142 patients over a 27 -month period was performed. Cohorts were studied according to underlying pathology, referring specialty and intended duration of stent placement. Measured outcomes were time to stent removal or stent exchange, death, forgotten stents, and complications.

\section{Results}

The overall technical success rate of antegrade stent insertion was $98 \%$. Follow-up data was available for 145 successful procedures in 138 patients. Malignancy (47\%) and stone disease (35\%) were the commonest indications. Overall, 43 patients (31\%) died over a median follow-up period of 2.2 years. 29 of 64 patients $(45 \%)$ with malignancy died with stents in situ after a median interval of 3.5 months. Malignancy and unclear intended duration of stent placement were predictors of death with a stent in situ. Twelve patients $(9 \%)$ had forgotten stents and a strong association with gynecological malignancy was noted, which is felt to represent inadequate follow up of patients with non-urological pathology. Complications were reported in 13 patients (9\%), including ten cases of heavy stent encrustation and one malpositioned stent.

\section{Conclusions}

Prognostic factors should be considered in the management of patients stented for malignant obstruction, which is usually a marker of advanced disease. The hazards of inadequate follow up are highlighted, causing delays in stent removal and exchange, or the forgotten stent. Interventions are described to minimize these risks.

Keywords: Ureteric stent, Ureteric obstruction, Forgotten stent, Antegrade stent, Malignant obstruction 
Ureteric stents are commonly used to relieve upper urinary tract obstruction and can be inserted via an endoscopic retrograde or percutaneous antegrade approach. Percutaneous nephrostomy and antegrade ureteric stent insertion may be particularly advantageous in the context of pelvic malignant obstruction where the retrograde approach is likely to be less successful. ${ }^{1,2}$

Urologists are well aware of the perils of the forgotten ureteric stent and take responsibility for the appropriate follow up of endoscopically inserted stents with the aid of local stent registries. ${ }^{3}$ In the UK antegrade ureteric stents are inserted primarily by interventional radiologists without necessarily the involvement of a urologist as direct referrals can be made from other specialties. Stents are inserted for a range of indications and often several specialties such as oncology, gynecology, colorectal surgery, and nephrology are involved in the care of these patients. This presents potential difficulties in the appropriate follow up of patients with ureteric stents, with associated risk of delayed intervention and complication.

Previous studies have described the effectiveness of antegrade ureteric stent placement. ${ }^{1,2,4}$ However, to our knowledge there have been no large published studies to specifically evaluate the follow up of patients after antegrade ureteric stent placement and in particular the rate of forgotten stents in this group of patients. This study reviews indications and outcomes of antegrade stent procedures at one university hospital to provide prognostic data that may improve the management of these patients.

\section{PATIENTS AND METHODS}

A retrospective search using hospital procedure codes was performed to generate a list of consecutive antegrade ureteric stent procedures performed by interventional radiologists at one large UK University teaching hospital over a 27-month period between April 2015 and June 2017. Institutional approval was obtained for the study (Reference CA16418).

Electronic patient and radiology records were interrogated to exclude incorrectly coded procedures and gather patient demographic data including indication for stent insertion, specialty requesting the procedure, success of procedure and documentation of intended duration of stent placement. An initial analysis was carried out in September 2017 and this was updated in May 2018. Follow-up data was analyzed to determine the primary outcomes of stent removal or exchange and time to death. The number of forgotten stents and recorded complications were measured. Forgotten stents were defined as those that had been left in situ for over 12 months or for over six months with no documented plans for their removal or exchange in alive patients at the time of initial analysis.

Statistical analysis was carried out using IBM $^{\circledR}$ SPSS ${ }^{\circledR}$ Statistics version 24 with $P$ values $<0.05$ taken to indicate statistical significance. Non-parametric tests including chi-square test of independence and Spearman's rho were used to identify predictors of the outcomes of forgotten stent, overall mortality, death with stent and stent-related complications. Logistic regression was carried out using the forward stepwise (likelihood ratio) method. For survival analysis censoring was carried out for patients alive at the final analysis. Median survival time was estimated by the Kaplan-Meier method and median follow-up time estimated using the reverse Kaplan-Meier method.

For individual stent-related events such as time to stent removal or exchange, forgotten stent and stentrelated complications, each stent procedure was treated as a separate episode. For patient-specific outcomes of survival and death with stent, analysis was carried out on an individual patient basis. For patients with multiple stent procedures the time from first stent placement to the time of death or last follow-up was used for analysis.

\section{RESULTS}

Figure 1 outlines the patients and procedures included in the study. Stent procedures comprised of a mixture of inpatient and outpatient requests for urgent and elective indications with an overall median wait time from request to procedure of 6 days (interquartile range: 3-20 days). Table 1 summarizes the outcomes following successful stent procedures.

J Endolum Endourol Vol 1(2):e27-e33; November 13, 2018.

This article is distributed under the terms of the Creative Commons Attribution-Non

Commercial 4.0 International License. (2018 Raju et al. 


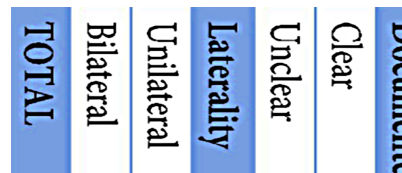
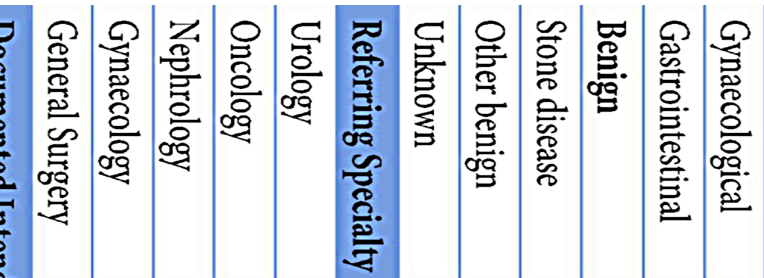

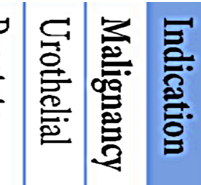

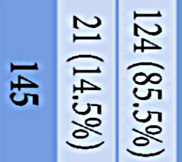

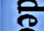

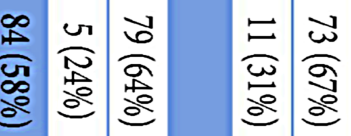

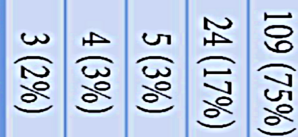

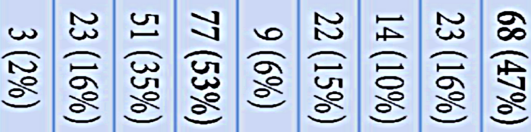

官望管

蛋

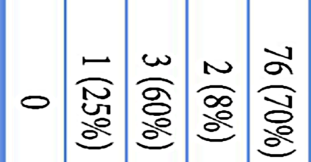

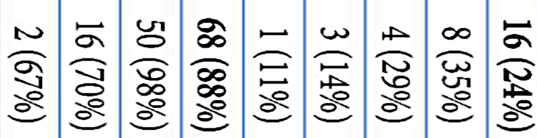

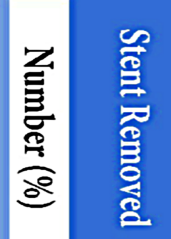

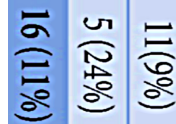

灾

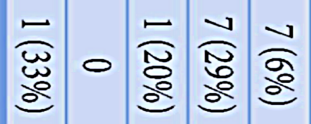

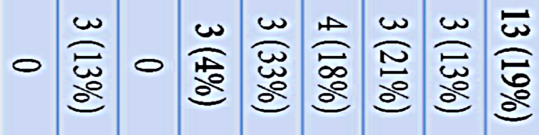

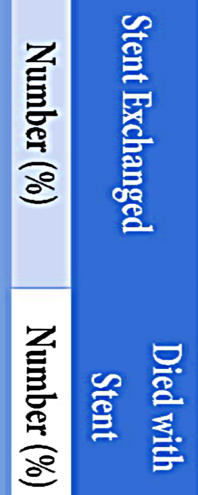

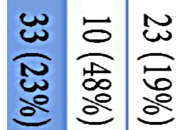

荅岕

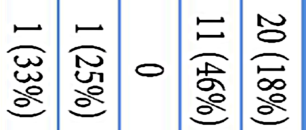

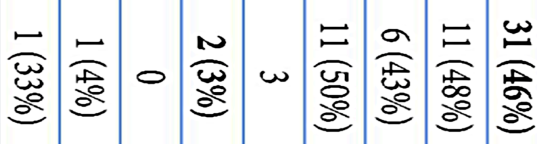

整高

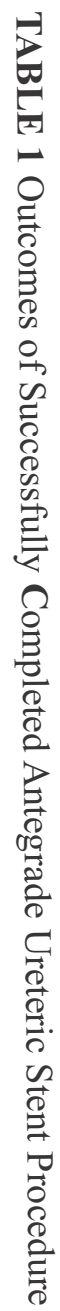

营 0 离

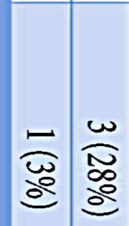

$0.00 \frac{\frac{a}{0}}{\frac{\pi}{n}}$

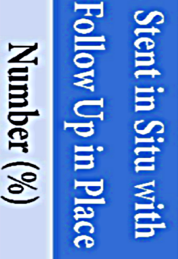

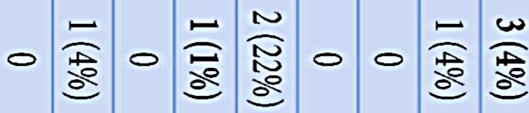

\section{+}

$+\quad-\quad+\quad$

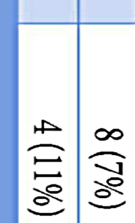

両

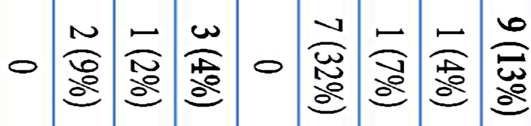

Z

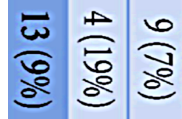

\begin{tabular}{c|c}
$\infty$ & 0 \\
$\frac{0}{a}$ & $\frac{0}{a}$
\end{tabular}

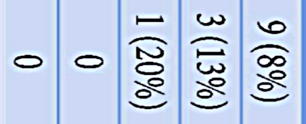

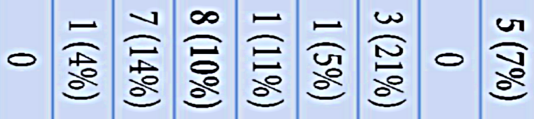

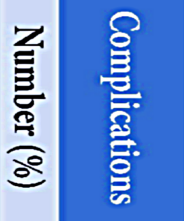


FIG. 1 Flow diagram of patients in study.

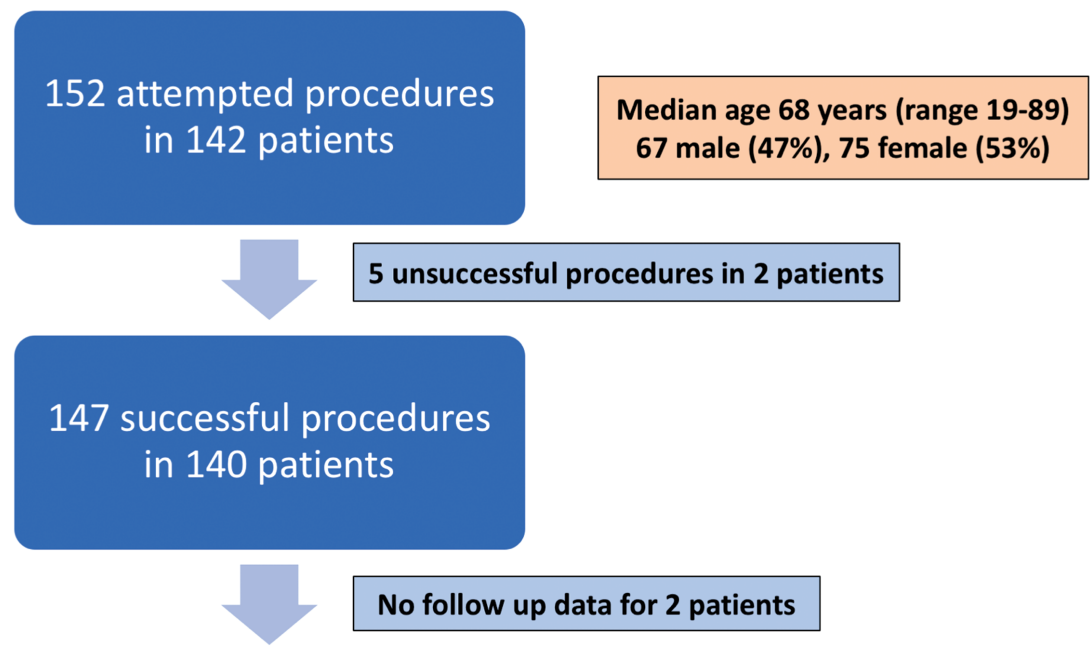

145 successful procedures

in 138 patients with follow

up data
Overall success rate $=98 \%$

Follow up rate $=\mathbf{9 7} \%$
Overall, $58 \%$ of stents were removed after a median interval of 61 days (range 1-299 days). 11\% of stents were exchanged after a median interval of 232 days (range 51-489 days), representing those that were intended to be long term indwelling stents. The majority $(81 \%)$ of the exchanged stents were inserted for malignant obstruction. Two patients waited over 12 months for stent exchange and these were therefore considered as forgotten stents.

Forty-three of 138 patients (31\%) died over a median follow-up period of 2.2 years. Twelve patients (9\%) died after their stents were removed and 31 (22\%) died with stents in situ. Median time from stent insertion to death was 7.9 months (240 days, range 6-768 days). Seven patients (5\%) died with stents that had been in situ for over a year. Figure 2 illustrates deaths in the malignant and benign obstruction cohorts and associated predictive factors. Of the 64 patients with malignant obstruction, 15 (23\%) survived with stents in situ for over a year.

Twelve patients $(9 \%)$ were deemed to have had forgotten stents, nine of whom had stents in situ for over a year. If patients who had died prior to the date of initial analysis with stents in situ for over 12 months are included this figure increases to $13 \%$. Figure 3 outlines the forgotten stents, complications and associated predictive factors for each. Thirteen stent-related complications (9\%) were reported, most commonly heavy stent encrustation in ten patients. Seven of the heavily encrusted stents had been in situ for over six months. However, in three patients with stone disease the stents had been in place for less than three months. One case of a malpositioned stent was reported, which required ureteroscopic replacement. In one procedure a safety string was left attached to the stent, which subsequently got encrusted and caused difficulties in stent removal. In two cases it was not possible to re-insert a stent cystoscopically during planned stent exchange.

\section{DISCUSSION}

Percutaneous nephrostomy and antegrade stent insertion is an established means of managing ureteric obstruction with a success rate of over $90 \%{ }^{1,2}$ The 
FIG. 2 Deaths in patients stented for malignant and benign obstruction.

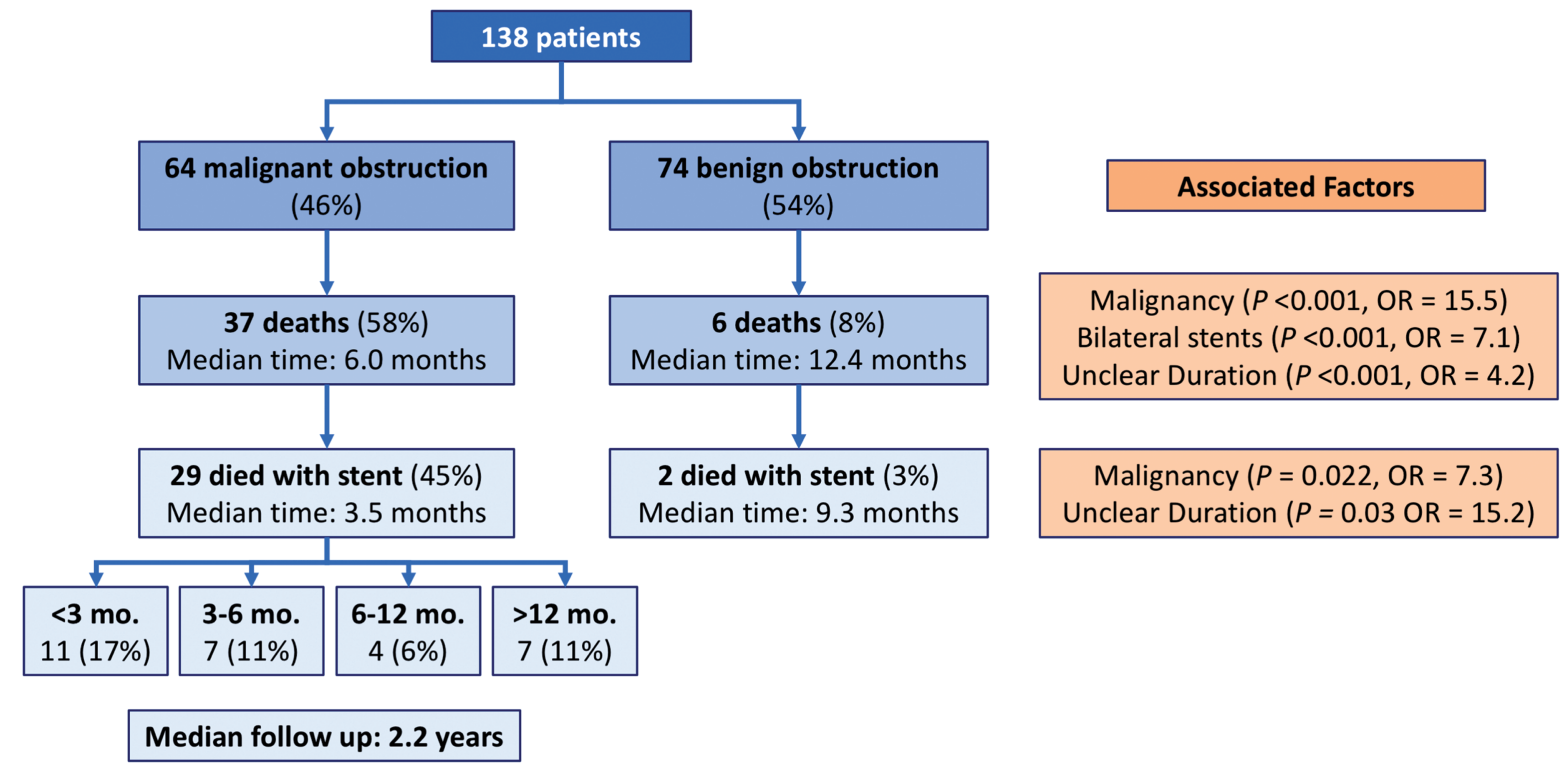

FIG. 3 Forgotten stents and complications.

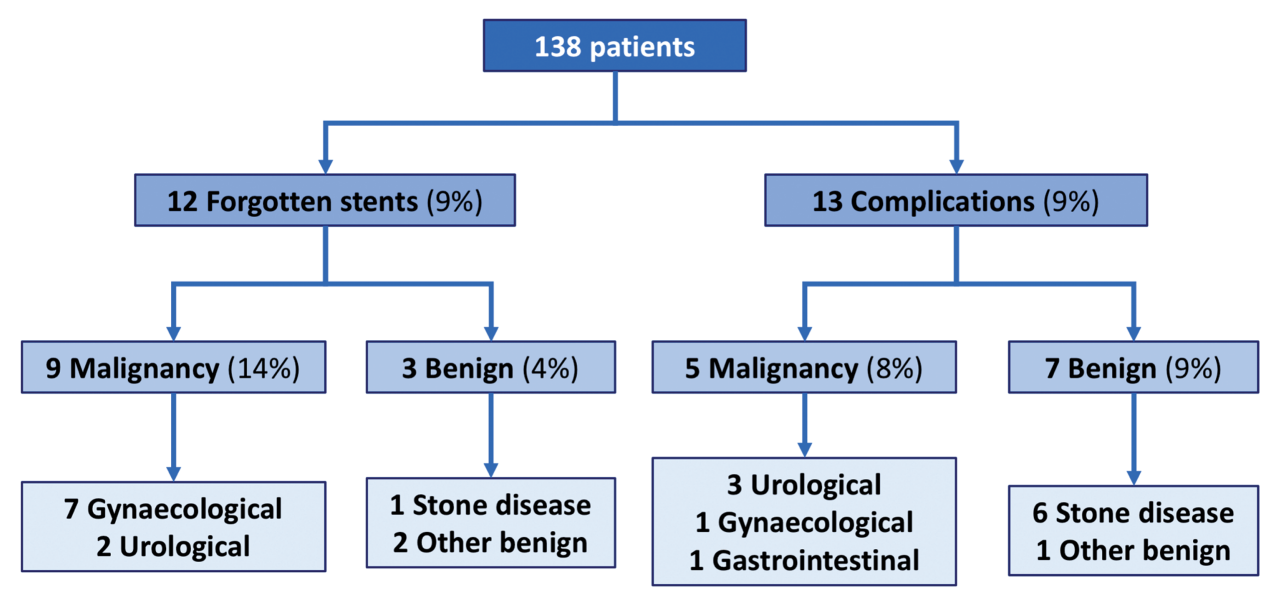

technical success rate in our series compares favourably to the existing published data.

Malignant obstruction and urolithiasis made up the indications for stent placement in over $80 \%$ of procedures. The fact that nearly half of the patients stented for malignancy died with original stents in situ within a short interval ( 3.5 months) is a reflection of the advanced stage of disease ${ }^{5}$ and should provide useful prognostic information when planning management of these stents. However, we must bear in mind that almost a quarter of patients with malignant obstruction survived with stents in situ for over a year. This poses the challenge of ensuring that the group of patients with favourable prognosis have timely management of their 
ureteric stents. Prognostic factors for urinary diversion in malignant ureteric obstruction have been described ${ }^{6}$ and could be helpful in identifying patients who may not benefit from ureteric stenting in the first instance and those who are likely to require long term stent management. Our study has highlighted requirement for bilateral ureteric stents as a poor prognostic factor.

Antegrade stents in the UK are primarily inserted by interventional radiologists following referrals from a range of different specialties and urologists are subsequently involved in management of these patients. The question therefore arises as to who is ultimately accountable for these patients' stents. The $9 \%$ rate of forgotten stents in our study represents a failure in appropriate follow up of this group of patients. Urologists will invariably need to be involved in the care of these patients if their stents are to be removed or replaced. Of the 12 patients with forgotten stents it was apparent that timely formal urology follow-up occurred in only two cases. The majority of forgotten stents were inserted for gynecological malignancy, which we have shown to be a significant associated factor. These patients had oncological follow up and the lack of a urologist's involvement may have contributed to the issue of stent management being neglected. It is also possible that in these cases patient survival was longer than initially anticipated and therefore referral for stent management was not felt to be appropriate. The relevance of a clear management plan for patients' stents was highlighted by our finding of the association between unclear documentation of intended stent duration and the odds of death with stent in situ, although we were unable to demonstrate an association with forgotten stents.

Urologists are cognisant of the risks of forgotten stents that are inserted endoscopically including encrustation, hematuria, infection and stent fracture, necessitating complex endoscopic and percutaneous procedures to manage these complications. ${ }^{3,7} \mathrm{Com}$ plications of antegrade ureteric stent insertion have also been reported including ureteric perforation and malpositioning. ${ }^{1,2,8}$ In our study stent encrustation was the most common reported complication and we were unable to demonstrate a clear association with stent dwell time. The only significant risk factor was bilateral stent insertion in the malignant obstruction cohort.

Despite the retrospective nature of this observational study, the clearly defined stent-related endpoints and high follow-up rate allow us to be confident in the robustness of the primary outcome data. The relatively small number of forgotten stents and complications may preclude reliable statistical analysis and we cannot therefore reach strong conclusions about predictive factors for these secondary outcomes.

After the initial analysis the cases of the patients with potential forgotten stents were reviewed and the appropriate clinical teams informed. As a result of this intervention, at the subsequent analysis four of these patients had undergone stent exchange and the remaining patients were awaiting review to plan management. Changes have also been implemented by the interventional radiology department to provide referring clinicians and patients with additional information at the time of stent insertion, advising them of the need to ensure follow up with a urologist for stent management. We anticipate that this will reduce the rate of forgotten stents and resulting complications in this cohort of patients.

\section{CONCLUSIONS}

The overall success rate of $98 \%$ in our series confirms the antegrade approach to be a highly effective method of ureteric stent placement. Ureteric obstruction in the context of malignancy is usually a sign of advanced disease and prognostic factors should be considered when deciding on the appropriateness of ureteric stenting and in planning management of stented patients. Robust systems are necessary to ensure stented patients are followed up by a urologist to minimize the risk of forgotten stents and the associated complications.

\section{FUNDING}

Not applicable.

\section{ACKNOWLEDGEMENTS}

We would like to thank Helen Thursby for assistance with data collection.

J Endolum Endourol Vol 1(2):e27-33; November 13, 2018.

This article is distributed under the terms of the Creative Commons Attribution-Non

Commercial 4.0 International License. (C2018 Raju et al. 


\section{REFERENCES}

1. van der Meer RW, Weltings S, van Erkel AR, et al. Antegrade ureteral stenting is a good alternative for the retrograde approach. Curr Urol 2017;10:87-91. 2017/05/30. DOI: 10.1159/000447157.

2. Hausegger KA and Portugaller HR. Percutaneous nephrostomy and antegrade ureteral stenting: techniqueindications-complications. Eur Radiol 2006;16: 2016-30. 2006/03/18. DOI: 10.1007/s00330-005-0136-7.

3. Monga M, Klein E, Castañeda-Zúñiga WR, et al. The forgotten indwelling ureteral stent: a urological dilemma. J Urol 1995;153:1817-19.

4. Hsu L, Li H, Pucheril D, et al. Use of percutaneous nephrostomy and ureteral stenting in management of ureteral obstruction. World J Nephrol 2016;5:172-81. DOI: 10.5527/wjn.v5.i2.172.
5. Kouba E, Wallen EM and Pruthi RS. Management of ureteral obstruction due to advanced malignancy: optimizing therapeutic and palliative outcomes. J Urol 2008;180:444-50. 2008/06/14. DOI: 10.1016/j.juro.2008.04.008.

6. Cordeiro MD, Coelho RF, Chade DC, et al. A prognostic model for survival after palliative urinary diversion for malignant ureteric obstruction: a prospective study of 208 patients. BJU Int 2016;117:266-71. 2015/05/24. DOI: 10.1111/bju.12963.

7. Sohrab A, Aneesh S, Sureka SK, et al. Forgotten Reminders: an Experience with Managing 28 Forgotten double-J stents and management of related complications. Indian J Surg 2015;77:1165-71. 2015/01/24. DOI: $10.1007 / \mathrm{s} 12262-015-1229-4$.

8. Rao AR, Alleemudder A, Mukerji G, et al. Extra-anatomical complications of antegrade double-J insertion. Indian J Urol 2011;27:19-24. DOI: 10.4103/0970-1591.78408.

J Endolum Endourol Vol 1(2):e27-e33; November 13, 2018.

This article is distributed under the terms of the Creative Commons Attribution-Non

Commercial 4.0 International License. (2018 Raju et al. 